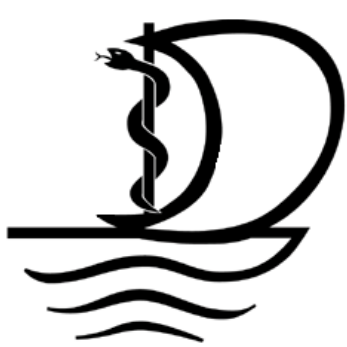

Deutsche Gesellschaft für Maritime Medizin e. V. www.maritimemedizin.de

\title{
Liebe Mitglieder der DGMM,
}

dies ist die erste Ausgabe der FTR nach unserer Mitgliederversammlung am 25.01.2013. Dieses Datum markiert einen Einschnitt in der Geschichte unserer Gesellschaft insofern, als unser langjähriger Vorsitzender, Bernd Schepers, auf eigenen Wunsch nicht mehr für den Vorstand kandidierte. Es war für uns alle nicht leicht, diesen Wunsch zu akzeptieren und durchaus eine Herausforderung, in dieser Situation die Kontinuität unserer Vereinsarbeit zu sichern. Schließlich gab es aber eine erfreuliche Zahl von Mitgliedern, die sich zu einer Kandidatur bereit erklärten, und es gelang, die Lücke zu schließen und die Reihen neu zu ordnen.

Das Ergebnis der Wahl vom 25.01. sehen Sie auf dem Foto unten. Der Vorstand hat inzwischen schon zweimal getagt und sich dabei eine anspruchsvolle Themenliste gestellt. In Zukunft wollen wir an dieser Stelle über die Dinge berichten, die uns in unserer Vorstandsarbeit beschäftigen, um Sie alle beteiligt zu halten und zu motivieren, mit Ihrem Vorstand in Dialog zu treten!

Heute ,senden' wir allerdings noch einmal die Botschaft, die unser langjähriger Vorsitzender Bernd Schepers am 25. Januar an die Mitgliederversammlung gerichtet hat - für alle, die nicht dabei sein konnten.

Mit herzlichen Grüßen

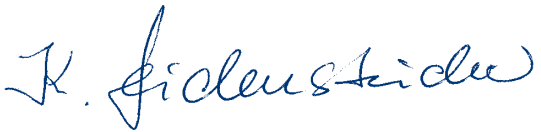

Klaus H. Seidenstücker, Tarp

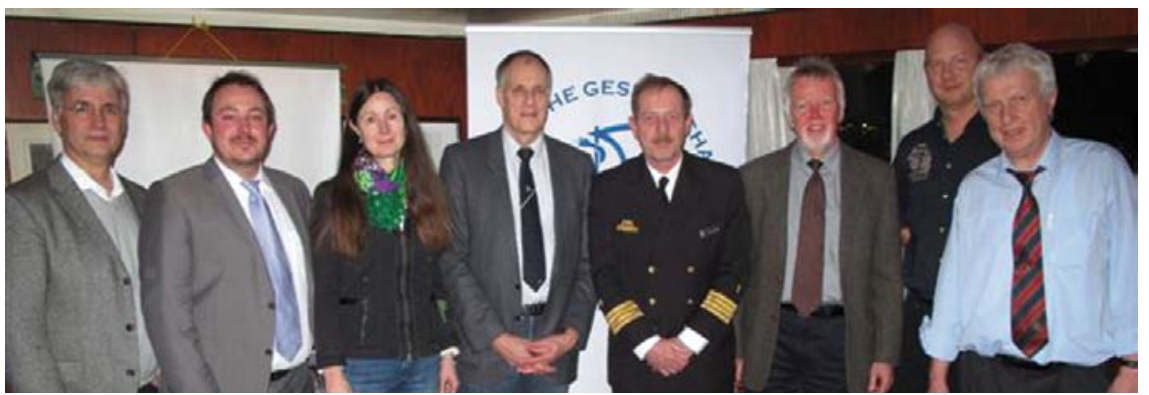

Der neu gewählte Vorstand der DGMM v.l.: Dr. Phillip Langenbuch, Dipl.-Ing. Christoph Sevenich, Dr. Eva-Maria Wichtmann, Dr. Jens Kohfahl, Dr. Stefan Neidhardt, Dr. Klaus Seidenstücker, Dr. Tim Lammerding sowie der ehemalige Vorsitzende Dr. Bernd Schepers.

\section{DGMM - Rückblick und Ausblick}

Liebe Mitglieder und Freunde der DGMM, anlässlich meines Ausscheidens aus dem Vorstand der DGMM - nachdem ich die Funktion des Vorsitzenden inzwischen seit circa 17 Jahren innehatte und vorher bereits 6 Jahre seit Gründung der DGMM stellvertretender Vorsitzender war, also nach circa 23 Jahren an der Spitze der DGMM - erlauben Sie mir einige Sätze in der Gesamtschau, als Standortbestimmung sowohl mit Blick zurück, wie auch mit Blick nach vorn und als Botschaft für die Zukunft aus meiner Sicht vorzutragen: In den ersten Jahren nach Gründung der DGMM 1990 ging es ja zunächst darum, die Gesellschaft zu etablieren und nach innen zu konsolidieren. In einem Umfeld, in dem sowohl insbesondere die zivile Abteilung für Schifffahrtsmedizin, aber auch das Schifffahrtmedizinische Institut der Marine vor essenziellen Herausforderungen standen und es zudem galt, die Schifffahrtsmedizin der ehemaligen DDR zu integrieren, sollte unserem Fachgebiet eine Stimme gegeben werden. Exemplarisch wurden schifffahrtsmedizinische Inhalte zunächst im Rahmen der ,Kieler Woche Workshops', später im Rahmen der Hamburger und Bremer Workshops und Kolloquien behandelt und in die 
DGMM-Mitgliedschaft sowie im begrenzten Rahmen auch in die Öffentlichkeit getragen. Dieser Informationsfluss wurde dann mit zahlreichen weiteren maritimmedizinischen Veranstaltungen, mit unserem DGMM-Mitteilungsblatt, Maritime Medizin' wie auch unserer DGMMHomepage fortgesetzt und weiter verbessert.

Erste Versuche, auch nach außen richtunggebend zu wirken, wurden beispielsweise mit der DGMM-Arbeitsgruppe ,Maritime Notfallmedizin', mit dem DGMM-Beitrag zum Zertifikat ,Maritime Medizin', mit dem Workshop und dem Curriculum ,Einführungslehrgang Maritime Notfallmedizin', mit der Beteiligung an den Sea-Cloud-Ärztetreffen und dem maßgeblichen DGMM-Beitrag zur Neufassung der sogenannten Richtlinie Nr. 4 ,Aufnahme einer Tätigkeit als Schiffsarzt/ Schiffsärztin' unternommen.

Die Erarbeitung der DGMM-Empfehlung zu den Offshore-Eignungsuntersuchungen war dann ein weiterer großer Schritt auf dem Weg zu einer medizinischen Fachgesellschaft, die ihr Fachgebiet wesentlich prägt.

Mit den ,Emder Workshops zur Offshoremedizin', mit der ,Fachtagung Kreuzfahrtmedizin' wie auch der eigenständigen Durchführung des Seminars ,Einführung in die Maritime Notfallmedizin' und auch durch die Kooperation mit der Zeitschrift Flugmedizin Tropenmedizin Reisemedizin des Thieme Verlags haben wir, hat die DGMM etliche neue Mitglieder gewonnen: Inzwischen gehen wir auf stattliche 230 ordentliche Mitglieder zu - das darf uns mit Stolz erfüllen und unterstreicht die zunehmende Bedeutung der DGMM!

Infolge unserer weiter entwickelten Publikationsaktivitäten wird die DGMM mehr und mehr wahrgenommen, unser Bekanntheitsgrad nimmt ständig zu, vorrangig im Inland, aber auch im benachbarten Ausland. Die in den vergangenen fast 23 Jahren von uns aufgegriffenen Fragestellungen haben auch gezeigt, wie viel noch unbearbeitet ist und welcher Forschungs- und Weiterentwicklungsbedarf noch auf die Maritime Medizin - sowohl national wie international - wartet. Dies zeigen auch immer wieder die alle 2 Jahre stattfindenden International Maritime Health Symposien (ISMH). Hier können die nationalen maritimmedizinischen Gesellschaften für die internationale Ebene wichtige Impulse geben.
Seefahrt ist international und damit ist auch für die Maritime Medizin die internationale Ebene immer entscheidender geworden. Ich selbst habe diesen Kontakt - zur IMHA und den ISMH - immer gepflegt und habe mich mit zahlreichen Aktivitäten auch immer wieder international eingebracht. Ich wünsche mir natürlich für die Zukunft weiterhin von deutscher Seite, von Seiten der DGMM eine Fortsetzung derartiger Aktivitäten!

Die Maritime Medizin steht sowohl national wie international weiterhin vor neuen und großen Aufgaben. Es liegt an uns, diese wahrzunehmen und die Themen voran zu bringen - qualitativ so hochwertig wie möglich und mit internationaler Vorbildfunktion und Ausstrahlung - wie es, so denke ich, von einer Fachgesellschaft mit zunehmender Bedeutung, wie es von der DGMM auch zunehmend erwartet wird. Sowohl im Vorstand, wie in unseren Arbeitsgruppen oder Projekten brauchen wir dafür die Bereitschaft zum Engagement mit Herzblut, mit der Motivation zum eigenen Beitrag. Wir brauchen die Bereitschaft zum offenen Wort, zum fairen Umgang miteinander und zum Teilen von Information, um uns auch in Zukunft bestmöglich für die gesundheitlichen Belange der Menschen auf See einzusetzen! Wenn wir gemeinsam diese Herausforderungen annehmen und befördern - wenn wir das Feuer weitertragen - dann steht der DGMM eine gute Zukunftsperspektive bevor. Das wünsche ich der DGMM! Packen wir es weiterhin an - und freuen uns darauf!

Abschließend bedanke ich mich bei allen DGMM-Mitgliedern für das Vertrauen und die Unterstützung, die Sie mir über nahezu 23 Jahre hinweg entgegengebracht haben!

Selbstverständlich bleibe ich der DGMM verbunden und werde auch in Zukunft der DGMM, insbesondere dem neuen Vorstand, gern mit Rat und Tat zur Seite stehen.

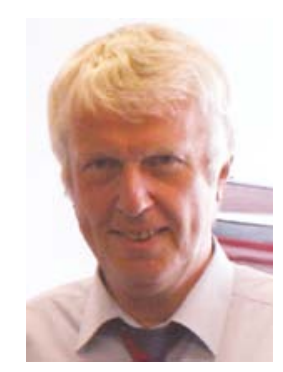

Vielen Dank!

Dr. Bernd-Fred Schepers 